Supporting Information For

\title{
State Transitions and Crystalline Structures of A Single Polyethylene Chain: MD Simulations
}

\author{
Shengming Jiang,,$+\ddagger$ Yuyuan Lu, ${ }^{*, \dagger, \ddagger}$ and Chuanfu Luo,,,$+ \ddagger$
}

$\dagger$ State Key Laboratory of Polymer Physics and Chemistry, Changchun Institute of Applied Chemistry, Chinese Academy of Sciences, Changchun, 130022, P.R. China

$\ddagger$ University of Science and Technology of China, Hefei, 230026, P.R. China

E-mail: yylu@ciac.ac.cn; luocf@ciac.ac.cn 


\section{I.Simulation Model}

The All-Atom (AA) model of OPLS-AA force field ${ }^{1,2}$ for polyethylene (PE) are used in our simulations. The OPLS-AA force field includes both bonded and non-bonded potentials between atoms. The total potential energy $\left(U_{t o t}\right)$ is the sum of five sub-potentials:

$$
U_{t o t}=U_{r}+U_{\theta}+U_{\varphi}+U_{l j}+U_{c}
$$

where, the five terms on the right hand are related to the interactions of bond, angle, dihedral, van der Waals, and Coulomb, respectively.

(i) $U_{r}$ is the potential energy associated with the chemical bonds:

$$
U_{r}=\sum_{i=2}^{N} \frac{1}{2} k_{b}\left(r_{i}-r_{0}\right)^{2}
$$

where $r_{0}$ is the equilibrium bond length and $r_{i}$ is the bond length between adjacent atom $i$ and $i-1, k_{b}$ is the constant.

(ii) $U_{\theta}$ is the potential energy associated with the bond angles (bending potential):

$$
U_{\theta}=\sum_{i=3}^{N} \frac{1}{2} k_{\theta}\left(\theta_{i}-\theta_{0}\right)^{2}
$$

where $\theta_{0}$ is the equilibrium bond angle and $\theta_{i}$ is the bond angle between three adjacent atoms $i, i-1$ and $i-2 . k_{\theta}$ is the constant.

(iii) $U_{\varphi}$ is potential energy associated with the dihedral angles (torsional potential):

$$
U_{\varphi}=\sum_{i=4}^{N} \sum_{i=0}^{5} C_{n}(\cos \varphi)^{n}
$$

where $\varphi$ is the dihedral angle between the two planes form by four consecutive atoms $i, i-1$, $i-2, i-3$ and $C_{i}$ is the constant. Here the form of Ryckaert-Bellmans dihedral is adopted. 
(iv) $U_{l j}$ is the van der Waals potential in the Lennard-Jones form:

$$
U_{l j}=\sum_{i}^{N} \sum_{j \geq i+3}^{N} \epsilon\left[\left(\frac{\sigma}{r_{i j}}\right)^{12}-\left(\frac{\sigma}{r_{i j}}\right)^{6}\right]
$$

where $r_{i j}$ is the distance between atoms $i$ and $j . \epsilon$ and $\sigma$ are the constants. Here, the van der Waals interactions between 1-2, 1-3, and 1-4 bonded neighbors are excluded.

(v) $U_{c}$ is the Coulomb potential energy associated with two charged particles:

$$
U_{c}=\sum_{i}^{N} \sum_{j>i}^{N} f \frac{q_{i} q_{j}}{\epsilon_{r} r_{i j}}
$$

where $r_{i} j$ is the distance between pair atoms $i$ and $j$, and $q_{i}$ and $q_{j}$ are the corresponding charges, respectively. $\epsilon_{r}$ and $f$ are the constants. The charges for $\mathrm{C}$ atom and $\mathrm{H}$ atom are -0.12 e $\left(-0.18\right.$ e for those belong to $\mathrm{CH}_{3}$ unit at head or tail), +0.06 e respectively. The electric conversion factor $f$ and the he relative dielectric constant $\epsilon_{r}$ are $138.935485 \mathrm{~kJ}$. $\mathrm{mol}^{-1} \cdot \mathrm{nm} \cdot \mathrm{e}^{-2}$ and 1 respectively, which indicates a vacuum environment.

The values of all parameters and constants listed above can be found in the original paper of OPLS-AA force field. ${ }^{1,2}$

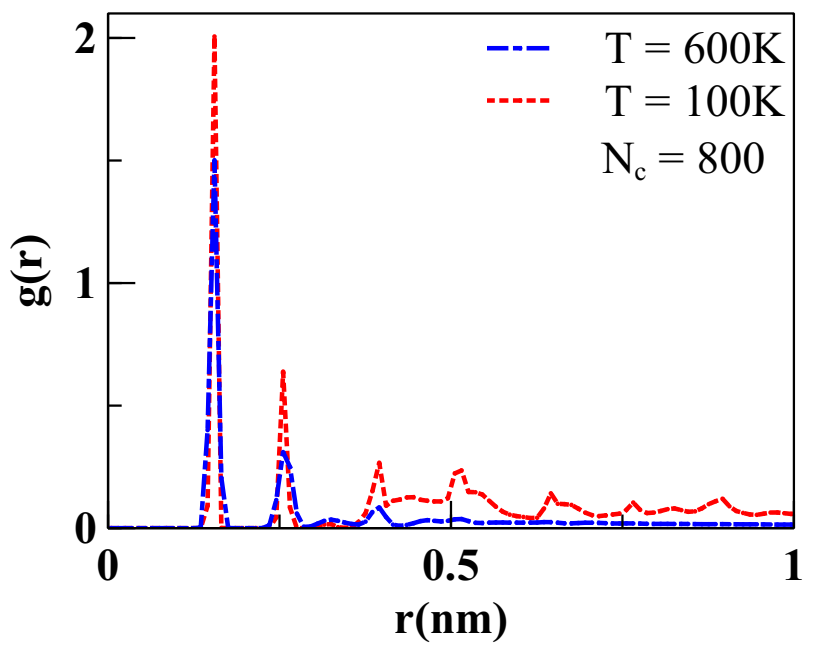

Figure S1: Pair correlation function $g(r)$ for a single chain with $N_{c}=800$ at $100 \mathrm{~K}$ (red) and $600 \mathrm{~K}$ (blue), respectively. 


\section{II.Pair correlation function}

Figure S1 shows the $g(r)$ of a long polyethylene chain at two different temperatures. At a lower temperature, the folded structure is formed, as indicated by the sharper peaks of $g(r)$ at $100 \mathrm{~K} .^{3}$

\section{III.Tight folding model}

We assume that the folded structure consists of thin stems with tight loops on the stem's head and tail, as shown in the inset of Figure 9(c) in main context. The average number of units per strand $\bar{n}_{\text {strand }}$ is given as $N_{c} / N_{\text {stem }}$, which is the sum of the unit numbers per stem $\bar{n}_{\text {stem }}$ and per loop $\bar{n}_{\text {loop }}$ :

$$
\bar{n}_{\text {strand }}=N_{c} / N_{\text {stem }}=\bar{n}_{\text {stem }}+\bar{n}_{\text {loop }}
$$

the contribution to $\lambda_{1}$ per unit in the stems and loops, $\lambda_{1}^{*}$, is given as:

$$
\lambda_{1}^{*}= \begin{cases}\bar{n}_{\text {stem }}^{2} l^{2} / 4 & \text { per loop unit } \\ \bar{n}_{\text {stem }}^{2} l^{2} / 12 & \text { per stem unit }\end{cases}
$$

where $l=0.127 \mathrm{~nm}$ is the $\mathrm{C}-\mathrm{C}$ bond length of the OPLS-AA model of polyethylene used in our simulations, and $\bar{n}_{\text {stem }} l$ represents the estimation of lamellar thickness given the crystal is large enough.

Then $\lambda_{1}$ is calculated by the summation of $\lambda_{1}^{*}$ over all single strands:

$$
\bar{n}_{\text {strand }} \lambda_{1}=\bar{n}_{\text {loop }} \frac{1}{4} \bar{n}_{\text {stem }}^{2} l^{2}+\bar{n}_{\text {stem }} \frac{1}{12} \bar{n}_{\text {stem }}^{2} l^{2}
$$

We define $\mu$ as the number fraction of stem units, which is also considered as the crystallinity: 


$$
\mu=\frac{\bar{n}_{\text {stem }}}{N_{c} / N_{\text {stem }}}
$$

Then we obtain the Equation (9) in main context:

$$
\frac{\sqrt{\lambda_{1}}}{l}=\frac{N_{c}}{N_{\text {stem }}} \sqrt{\frac{1}{4} \mu^{2}-\frac{1}{6} \mu^{3}}
$$

\section{IV.Calculation of $\Delta \varepsilon$}

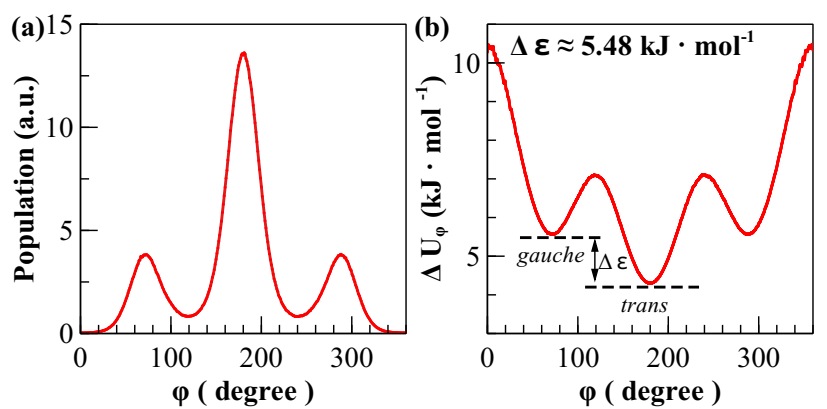

Figure S2: (a) Population of dihedral angles $\varphi$ for a single chain with $N_{c}=800$ at $600 \mathrm{~K}$. (b) potential energy of dihedral angle $\varphi$ obtained by using the inverse-Boltzmann relation. The energy difference between gauche and trans states is $\Delta \varepsilon$.

The potential difference between the gauche and trans states is represented by $\Delta \varepsilon$. A constant temperature simulation of a single chain with $N_{c}=800$ was performed at $600 \mathrm{~K}$ for $50 \mathrm{~ns}$ to obtain the distribution of dihedral angles, as shown in Figure S2(a). The inverse-Boltzmann relation was used here to calculate the equivalent energy of dihedral angle $U(\varphi) / k_{B} T$, which is shown in Figure $\mathrm{S} 2(\mathrm{~b})$. The value of $\Delta \varepsilon$ is calculated as $5.48 \mathrm{~kJ} \cdot \mathrm{mol}^{-1}$ $\left(1.28 k_{B} T\right.$ at $\left.600 \mathrm{~K}\right)$.

\section{V.Crystalline structure of bulk system using OPLS-AA}

For bulk PE systems using OPLS-AA model, the orthorhombic phase can be obtained from a cooling process from 600 to $100 \mathrm{~K}$ in $50 \mathrm{~ns}$ with a NPT ensemble $(1 \mathrm{~atm}$.). The orthorhombic 


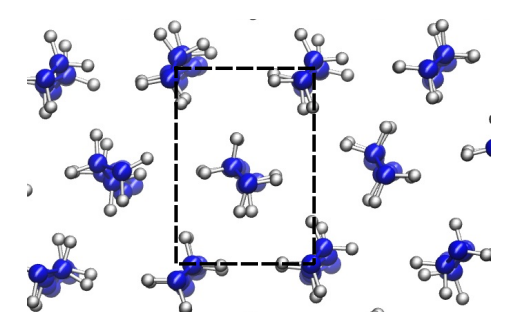

Figure S3: The crystalline structure (orthorhombic phase) of a bulk system (10 PE chains with $N_{c}=500$ ) obtained by using the same OPLS-AA model. Only one crystalline part is shown for clarity.

phase of a bulk system consisting $10 \mathrm{PE}$ chains with $N_{c}=500$ is shown in Figure S3.

\section{VI.Simulation details and results for long cut-off dis-}

\section{tances}
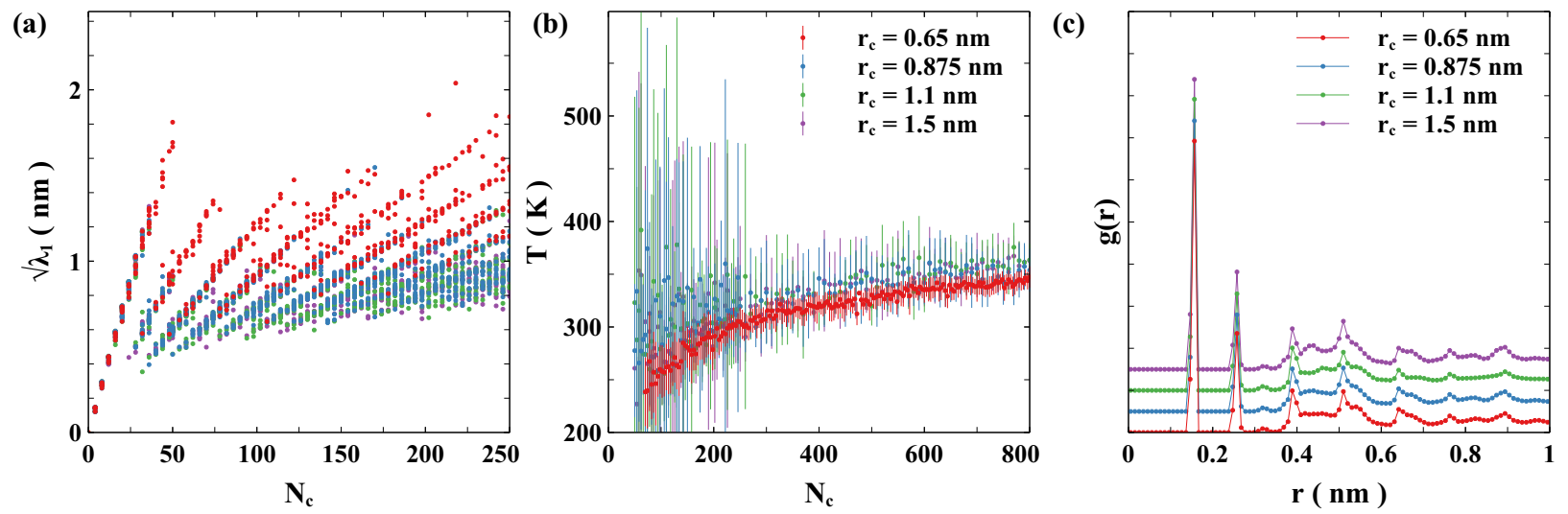

Figure S4: (a) Distribution of $\sqrt{\lambda_{1}}$ and (b) $T_{\text {fold }}$ versus $N_{c}$ with different cut-off distances. (c) the radial distribution function $g(r)$ of folding structure with different cut-off distances. Here, the values of $\sqrt{\lambda_{1}}$ for $r_{c}=0.65 \mathrm{~nm}$ are larger than those for longer cut-off distances as better crystalline structures are obtained (like the crystallization from solution always results in a very high crystallinity). However, the folding mode is the same and there is no qualitative difference and $T_{\text {fold }}$ is only slightly shifted. Please note that the number of samples for $r_{c}=0.65 \mathrm{~nm}$ are almost five times more than that for longer ones and we can obtain a better averaged value as the benefit of reduced computing cost.

The series of simulations with different chain length $\left(N_{c} \in\{2,6,10, \cdots 250,260,270 \cdots 800\}\right)$ and the temperature range, from $800 K$ to $100 K$ (70 ns), for continues cooling process. Here, the distribution of $\sqrt{\lambda_{1}}$ and the folding temperature $T_{\text {fold }}$ with different cutoff distance are 

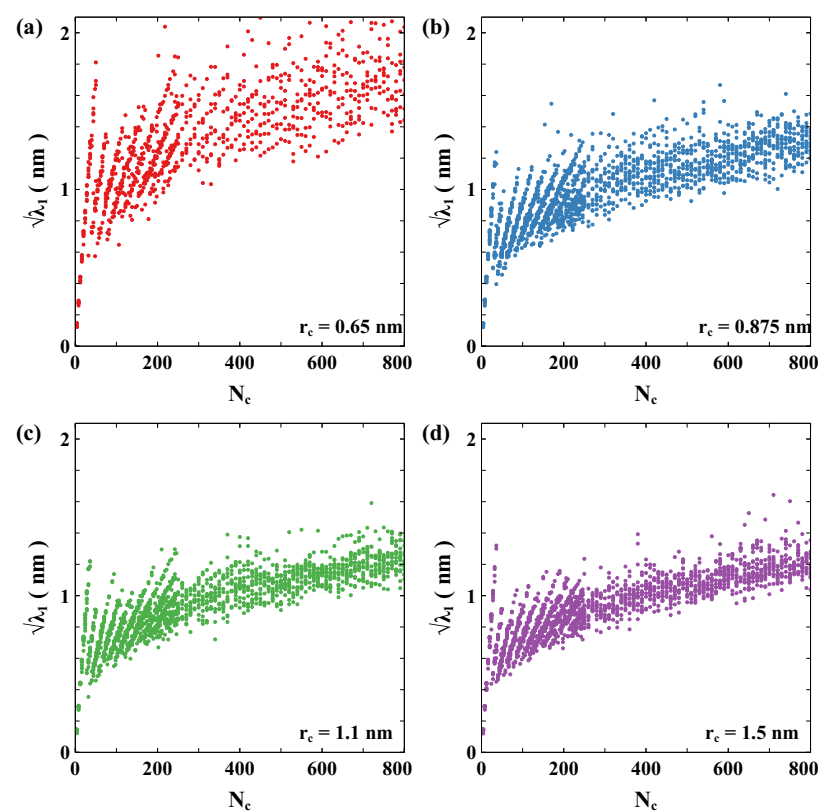

Figure S5: Distribution of $\sqrt{\lambda_{1}}$ versus $N_{c}$ with different cut-off distances (a) $r_{c}=0.65 \mathrm{~nm}$, (b) $r_{c}=0.875 \mathrm{~nm}$, (c) $r_{c}=1.1 \mathrm{~nm}$, and (d) $r_{c}=1.5 \mathrm{~nm}$, by using the same number of samples $\left(N_{c}=2,6,10, \cdots 250,260,270 \cdots 800\right)$.

shown in Figure S4 and Figure S5. Few folding structures with different cut-off distance are

shown in Figure S6. We notice that the $\sqrt{\lambda_{1}}$ increases with reduce of the cut-off distance, which is the result of screen effect. Thus the higher value of $\sqrt{\lambda_{1}}$ for shorter cut-off distance means higher crystallinity like the crystallization from polymer solution. However, we should emphasize that the folding mode is the same and there is no qualitative difference and $T_{\text {fold }}$ is only slightly shifted. For the highly fluctuated conformation of a single-chain, the distribution of a structural parameter is more meaningful than an average one.

\section{VII. $T_{1}$ vs. $T_{\text {collapse }}$ and $T_{2}$ vs. $T_{\text {fold }}$}

We plot the effective excluded volume $v$ varying with temperature $T$ for different chain lengths $N_{c}$ in Figure $\mathrm{S} 7(\mathrm{a})$. The temperature $T_{1}$ and $T_{2}$ is determinated by the turning points. Their relations with $T_{\text {collapse }}$ and $T_{\text {fold }}$ are shown in Figure $\mathrm{S} 7(\mathrm{~b})$. 


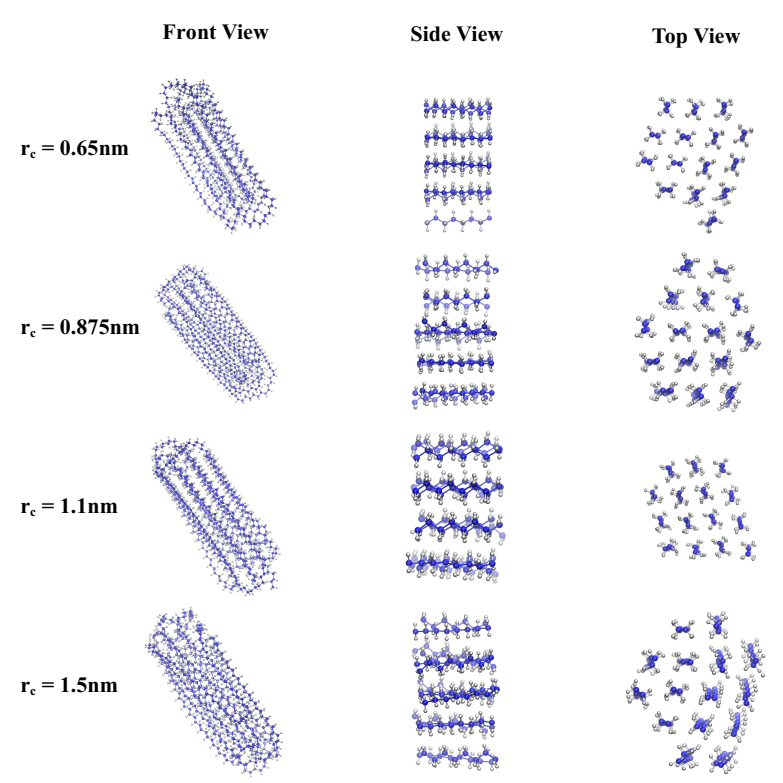

Figure S6: Snapshots of the folded structures of a single chain $\left(N_{c}=500\right)$ with different cut-off distances.
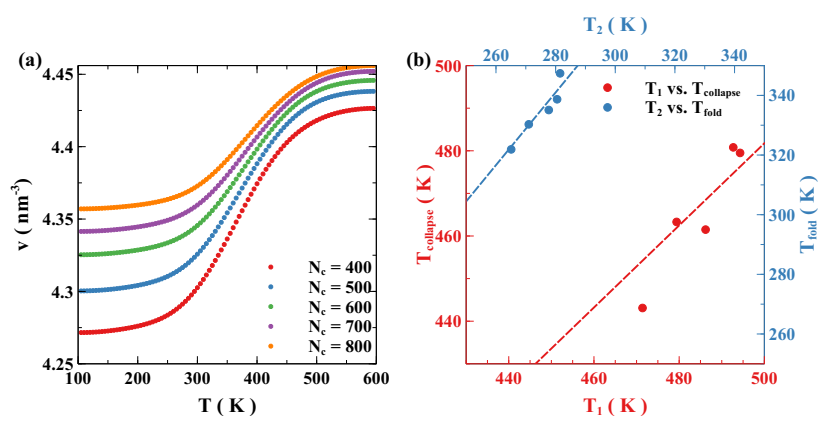

Figure S7: (a) Effective excluded volume $v$ versus temperature $T$ for different chain lengths $N_{c}$ with the cut-off distance $\left(r_{c}=0.65 \mathrm{~nm}\right)$. (b) Relation-plots of $\left(T_{1}, T_{\text {collapse }}\right)$ and $\left(T_{1}, T_{\text {fold }}\right)$. Here $T_{1}$ and $T_{2}$ are the turning points shown in (a). The slopes of red and blue dashed lines are $1.02,1.14$, respectively

\section{VIII.Extra snapshots for the single chain}

We plot the snapshots of two single chains $\left(N_{c}=200,300\right)$ during the cooling process with different chain lengths in Figure S8(a) and the snapshots of two long chains $\left(N_{c}=500,800\right)$ at $T=600 \mathrm{~K}$ with different cut-off distances $r_{c}$ in Figure S8(b). 
(a)

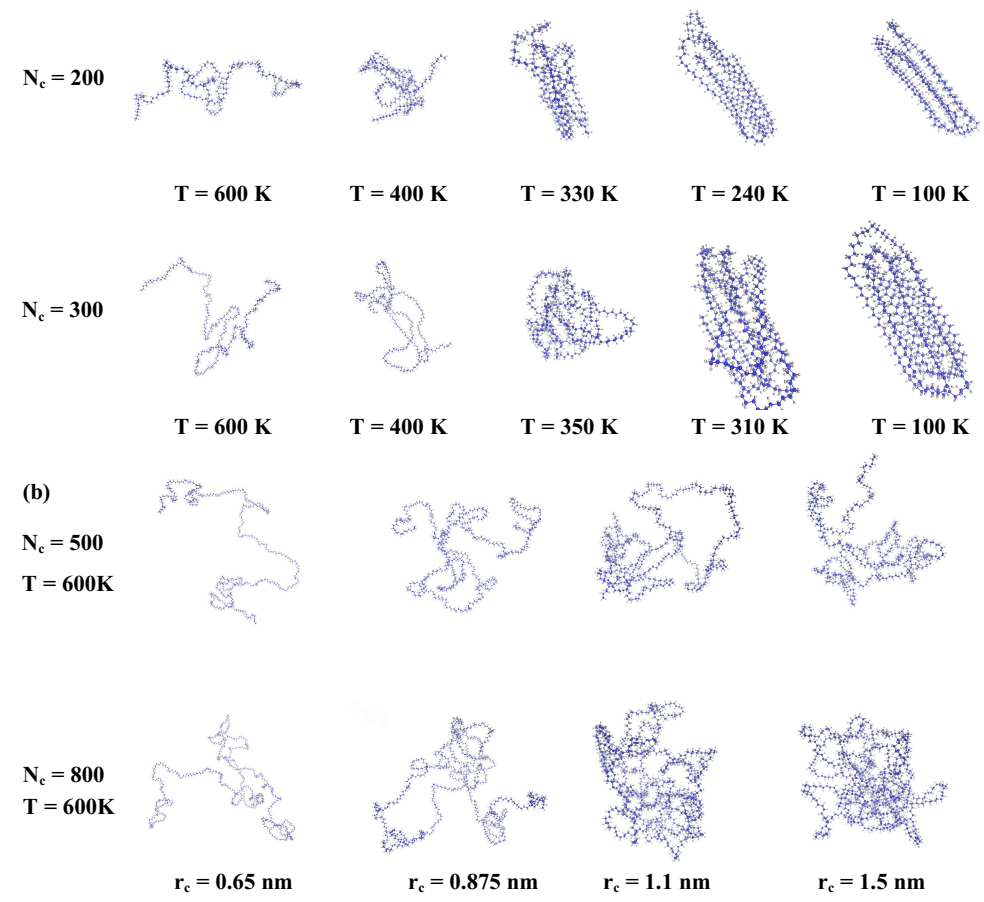

Figure S8: (a) Snapshots of the structures of single chains with various chain lengths $\left(N_{c}=200,300\right)$ during the cooling process. The largest radius of gyration tensor $\sqrt{\lambda_{1}}$ is approximate to $1.1 \mathrm{~nm}$. (b) Snapshots of structures of a single chain $\left(N_{c}=500,800\right)$ at $T=$ $600 \mathrm{~K}$ with different cut-off distances $r_{c}$.

\section{References}

(1) Jorgensen, W. L.; Madura, J. D.; Swenson, C. J. Optimized intermolecular potential functions for liquid hydrocarbons. J. Am. Chem. Soc. 1984, 106, 6638-6646.

(2) Jorgensen, W. L.; Maxwell, D. S.; Tirado-Rives, J. Development and testing of the OPLS all-atom force field on conformational energetics and properties of organic liquids. J. Am. Chem. Soc. 1996, 118, 11225-11236.

(3) Li, T.; Yang, X.; Nies, E. A replica exchange molecular dynamics simulation of a single polyethylene chain: temperature dependence of structural properties and chain conformational study at the equilibrium melting temperature. J. Chem. Theory Comput. 2011, 7, $188-202$. 